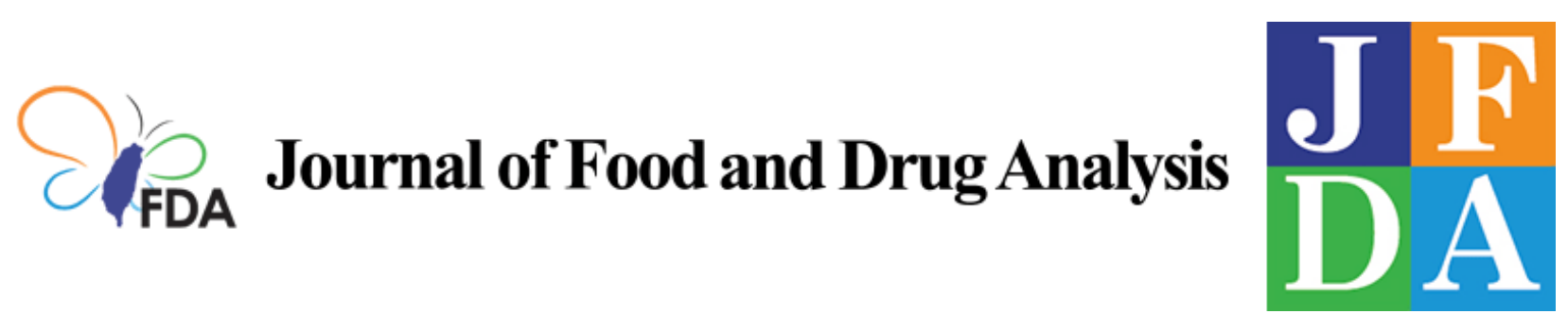

Volume 28 | Issue 4

Article 9

2020

\title{
Improved food additive analysis by ever-increasing nanotechnology
}

Follow this and additional works at: https://www.jfda-online.com/journal

Part of the Food Science Commons, Medicinal Chemistry and Pharmaceutics Commons, Pharmacology Commons, and the Toxicology Commons

(c) (i) (8)

This work is licensed under a Creative Commons Attribution-Noncommercial-No Derivative Works 4.0 License.

\section{Recommended Citation}

Zhang, Jing; Hu, Shui; Du, Yi; Cao, Ding; Wang, Guirong; and Yuan, Zhiqin (2020) "Improved food additive analysis by ever-increasing nanotechnology," Journal of Food and Drug Analysis: Vol. 28 : Iss. 4 , Article 9. Available at: https://doi.org/10.38212/2224-6614.1152

This Review Article is brought to you for free and open access by Journal of Food and Drug Analysis. It has been accepted for inclusion in Journal of Food and Drug Analysis by an authorized editor of Journal of Food and Drug Analysis. 
Improved food additive analysis by ever-increasing nanotechnology

Cover Page Footnote

Jing Zhang and Shui Hu contributed equally to this work. 


\title{
Improved food additive analysis by ever-increasing nanotechnology
}

\author{
Jing Zhang ${ }^{a, 1}$, Shui $\mathrm{Hu}^{\mathrm{a}, 1}$, Yi Du ${ }^{\mathrm{b}}$, Ding Cao ${ }^{a}$, Guirong Wang ${ }^{\mathrm{a}}$, Zhiqin Yuan ${ }^{\mathrm{a}, \mathrm{b}, *}$
}

\begin{abstract}
a State Key Laboratory of Chemical Resource Engineering, College of Chemistry, College of Material Science and Engineering, Beijing University of Chemical Technology, Beijing 100029, China

${ }^{\mathrm{b}}$ Department of Chemistry, Key Laboratory of Bioorganic Phosphorus Chemistry \& Chemical Biology (Ministry of Education), Tsinghua University, Beijing 100084, China
\end{abstract}

\begin{abstract}
Improper use of food additives may lead to potential threat to human health, making it important to develop sensitive and selective method for their detection. Nanomaterials with unique chemical and electrochemical properties show extensive applications in the design of food additive sensing systems. In this review, we summarize the recently adopted electrochemical and optical analysis of food additives based on nanomaterials. Detection of typical food additives (colorants and preservatives) by using different sensing mechanisms and strategies are provided. In addition, determination of illegal food additives is also briefly introduced. Finally, this review highlights the challenges and future trend of nanomaterial-based food analysis system.
\end{abstract}

Keywords: Nanomaterials, Food colorants, Food preservatives, Illegal additives, Detection

\section{Introduction}

A $\mathrm{s}$ the changing population and lifestyle, there are increasing requirements on food quality and texture. To enhance the appearance, fragrance, taste, or storage of food, additional ingredients, usually called as food additives, are generally applied in food industry. According to their functions, these food additives can be classified into different species, including colorants, preservatives, sweeteners, thickeners, emulsifiers, raising agents, and acidity regulators, etc [1]. Reasonable use of these food additives not only improves the color and taste of food, but also boosts the nutritional value. Unfortunately, improper use of some food additives leads to a potential threat to human life and health [2,3]. In addition, some food additives have been demonstrated as potential disease triggers. For example,
Lakshmi et al. found that indigo carmine colorant may cause permanent damage to the cornea and conjunctiva [4]. Ma et al. also reported that excessive intake of indigo carmine has mutagenic effects and may lead to tumors [5]. Furthermore, some synthetic chemicals with high toxicities have also been used as illegal food additives, which cause serious food accidents. Therefore, the development of simple and effective method to monitor the content of food additives is extremely important for protecting Human beings.

To accomplish successful detection of food additives, many methods based on liquid chromatography, mass spectrometry, and electrochemistry have been reported [6-12]. For instance, Harp et al. reported the determination of seven certified colorants using liquid chromatography [13]. In comparison with sole liquid chromatography technique, the combination of liquid chromatography and

Received 29 May 2020; revised 17 August 2020; accepted 1 September 2020.

Available online 1 December 2020

* Corresponding author at: State Key Laboratory of Chemical Resource Engineering, College of Chemistry, College of Material Science and Engineering, Beijing University of Chemical Technology, Beijing 100029, China. Fax: +861064411957.

E-mail address: yuanzq@mail.buct.edu.cn (Z. Yuan).

1 These authors contributed equally to this work. 
mass spectrometry provides higher sensitivity toward food additive analysis. Using this coupling technique, Hao et al. has proposed a sensitive and selective method for tertiary butylhydroquinone (typical preservative) detection [9]. Despite of the analysis of food additives using these conventional detection techniques, there are still some drawbacks, including complicated sample preparation, complicated analytical steps, and time-consuming testing. In addition, the sensitivities of some systems do not suffice the demand of food quality evaluation. And thus, the exploration of new systems with simplicity and high sensitivity for food additive is still charming.

Nanomaterials with large specific surface area and unique chemical/electrochemical properties are widely used as sensing probes, nanocatalysts, nanodrugs, and so on [14-20]. In last two decades, profiting from the ever-increasing nanoscience and nanotechnology, a number of facile and sensitive detection techniques toward food additives have been developed [21-23]. A brief summary of nanomaterial-based detection systems toward food additives would benefit the researchers to choose proper method in the future work. In this review, we essentially focus on recent advances in food additive analysis using nanomaterials that have been reported since 2016. Detection of two kinds of extensively used food additives, including colorants and preservatives, is discussed. In addition, the analysis of illegal food additives based on nanotechnology is also introduced. Finally, challenges and future outlooks of nanomaterial-based food additive analysis are discussed.

\section{Food colorant detection}

Food colorants control the aesthetic appearance of food, which makes food more attractive and plays an important role in the choice of food [24]. Thus, food colorants are essential for food manufacturing industry because they can excite the purchase interest of consumers. Many colorful compounds can be used as food colorants, including sunset yellow, allure red, lemon yellow, erythrosine, carmine, amaranth, tartrazine, acid orange, and so on [25-36]. In general, natural food colorants are harmless or even beneficial to the human body. Through the genotoxicity evaluation, Hobbs et al. found that gardenia blue as natural food colorant does not pose genotoxic concern [37]. Nevertheless, the high cost of natural colorants leads to the wide circulation of many bright colored synthetic chemicals with high stability and low cost. These synthetic colorants are usually organic reagents prepared by artificial chemical synthesis. They are mainly used in the coloring of carbonated drinks, fruit juice drinks, confectionery wine, confectionery, candy, hawthorn products, pickled vegetables, ice cream, jelly, chocolate, cream, instant coffee, and other foods. However, it should be noticed that many food colorants (especially synthetic food colorants) are suspected of carcinogenesis and have been strictly limited in use. To avoid food colorant contamination, a few methods have been reported by using nanomaeterials as signal reporters [38,39]. In view of the usage of these food colorants, analysis of them in soft drinks and other foods are concluded.

\subsection{Colorants analysis in soft drinks}

Soft drink is defined as the natural or artificial beverage with low alcohol content $(<0.5 \%$, mass ratio). In addition to the high cost, natural colorants are easy to change color or fade in the processing of soft drinks. Therefore, water-soluble synthetic colorants are usually used to improve the sensory quality of soft drinks, which increases the buyer's preference. The common soft drinks include carbonated drinks, juice drinks, vegetable juice drinks, tea drinks and bottled drinking water drinks, and so on. In order to achieve rapid and sensitive detection of artificial colorants in soft drinks, nanomaterials have been widely used as accumulators or optical reporters. For example, Bakheet et al. reported a fluorimetric Allura red detection system in soft drinks by using poly(ionic liquid) immobilized magnetic nanoparticles as the adsorbent [40]. In this report, the author adopted magnetic solid phase extraction technology, which greatly shortened the analysis time. Compared with conventional solidphase extraction fillers, this method only requires small amount of adsorbent and short equilibrium time to achieve low-concentration micro-extraction. Such high extraction capacity and extraction efficiency are majorly due to the large specific surface area and short diffusion distance of nanoparticles. Because of the good stability and hydrophobicity of polyionic liquids, the authors used free radical copolymerization to fix polyionic liquids (PILs) on the surface of magnetic nanoparticles $\left(\mathrm{Fe}_{3} \mathrm{O}_{4} @ \mathrm{SiO}_{2}\right)$ to obtain adsorbents $\left(\mathrm{Fe}_{3} \mathrm{O}_{4} @ \mathrm{SiO}_{2} @ P I L s\right)$. Moreover, Allura red can be firmly adsorbed on the PIL by $\pi-\pi$ hydrophobic dispersion and weak dipole interaction mechanism because of the azo and aromatic structures, which greatly improves the separation rate. Finally, magnetic solid phase extraction was combined with fluorescence spectrophotometry, they successfully achieved Allura red analysis under the optimized conditions, with a limit of 
detection (LOD) of $0.002 \mu \mathrm{g} / \mathrm{mL}$. And good linearity was observed in concentration range from 0.01 to $0.50 \mu \mathrm{g} / \mathrm{mL}$. Additionally, the proposed method could be applied for Allura red analysis in candies and beverages samples with acceptable deviations. The same is the principle of extraction. Swapnil Tiwari et al. in order to detect tartrazine in soft drinks, the silver nanoparticles were modified with hydrophobic ligands, and then the tartrazine was extracted by single drop microextraction. Finally, the satisfactory results were obtained by combining with diffusion reflection Fourier transform infrared spectroscopy [41].

As can be seen from the above reports, combining the excellent surface adsorption activity of nanoparticles with traditional detection methods is currently an effective method for detecting food colorants in soft drinks. Among the traditional detection methods, the electrochemical method has been widely used due to its portability, high sensitivity, low cost and short analysis time [42-46]. In this detection method, the glassy carbon electrode (GCE) has become a popular working electrode by virtue of its good conductivity and high chemical stability. As a kind of chemically modified electrode, it will be more and more popular to modify glassy carbon electrode with nanomaterials to improve its electrochemical performance. When choosing the materials to modify the GCE, carbon nanotubes have unique physical and chemical properties, which can promote the electron transfer of electroactive substances, and show better selectivity and more obvious electrocatalysis for the target compounds. Among the carbon nanotubes, multi-walled carbon nanotubes (MWCNTs) have outstanding tensile strength, excellent electrical conductivity, high chemical stability, and can produce good electron transfer rate. Compared with the bare electrode, their films have considerable surface enhancement effect, which can significantly improve the sensitivity of the sensor. For example, P. Sierra-Rosales et al. used 1,3-dioxolane as the dispersant and modified the glassy carbon electrode with MWCNTs to obtain an electrochemical sensor with high stability and good repeatability. It showed high sensitivity and reproducibility in the detection of tartrazine, sunset yellow and carmoisine in soft drinks [29]. After surface functionalization of MWCNTs modified electrode with molecularly imprinted polymer through the electropolymerization of acrylamide in the presence of sunset yellow template, Arvand et al. developed a selective electrochemical method for sunset yellow [47]. The good specificity is attributed to the molecular recognition of molecularly imprinted polymer, which rules out the interference of other chemicals. With the proposed electrochemical system, they realized sunset yellow sensing in the concentration range from 0.05 to $100 \mu \mathrm{M}$, with a LOD of $5.0 \mathrm{nM}$. Polydopamine with good electron transfer capability has been widely used as the surface coating material for electrodes. Using dopamine as the monomer, Yin et al. reported the surface functionalization of MWCNTs modified electrode with polydopamine (molecularly imprinted polymer) in the presence of sunset yellow template [48]. The modified electrode is capable of sunset yellow sensing with excellent specificity because of the highly matched imprinted cavities, as shown in Fig. 1. Surface coating of polydopamine not only enhances the capture of sunset yellow, but also promotes the electron transfer, which leads to high sensitivity. Using this electrode, electrochemical detection of sunset yellow was achieved in the concentration range of $2.2 \mathrm{nM}-4.64 \mu \mathrm{M}$, with a LOD of $1.4 \mathrm{nM}(\mathrm{S} / \mathrm{N}=3)$. This LOD is much lower than most previously reported works. The prepared sensor was successfully used to detect sunset yellow in real spiked samples. The combination of MWCNTs with gold nanoparticles (AuNPs) is an attractive material with a wide size range and size dependent optical, magnetic, electronic and chemical properties. Chitosan (CHIT) has good adhesion, high mechanical strength, excellent film-forming ability and nontoxic, and is easy to be chemically modified because of its rich amino groups. Rovina et al. Modified GCE with graphene (GO), MWCNTs and AuNPs via CHIT to obtain the electrode (CHIT/GO/MWCNTs/ AuNPs/GCE) [49]. The electrode was applied to the determination of sunset yellow in soft drinks in the concentration range of $10-90 \mathrm{mg} / \mathrm{mL}$, LOD of $0.032 \mathrm{mg} / \mathrm{mL}$, showing excellent sensitivity.

In addition to MWCNTs, other nanomaterials (bimetallic nanoparticles, transition metal oxides, graphene oxide, etc.) can also be used as good candidates for electrode modification and electrochemical activity enhancement $[27,30,36,50]$. For example, in order to detect amaranth in soft drinks, Wang et al. prepared a porous graphene materialgraphene nanomesh (GNM) to modify the glassy carbon electrode (GCE) to obtain GNM/GCE electrode [27]. In addition, in order to prove that this porous nanomaterial modified electrode has higher electrochemical performance, the author compared it with reduced graphene oxide (RGO) modified glassy carbon electrode (RGO/GCE) and bare glassy carbon electrode (GCE). Because the introduction of macroporous density into graphene sheet is not only conducive to the adsorption of amaranth growth molecules to the working electrode surface through the pores, but also conducive 
to the rapid diffusion of electrons in the electrochemical detection process, the electrochemical activity of GNM/GCE is significantly higher than that of RGO/GCE and bare GCE. In addition, Parisa S. Dorraji et al. synthesized a $\mathrm{ZnO} /$ cysteic acid nanocomposite to modify the glassy carbon electrode (GCE) and can be used to simultaneously determine sunset yellow and tartrazine in soft drinks [30]. In this report, Cysteine is formed by electrochemical oxidation of L-cysteine on the surface of glassy carbon electrode (GCE), and is used as a suitable polymer framework to make $\mathrm{ZnO}$ nanofilms uniformly deposited on the electrode surface. The fabricated modified electrode facilitated the electron transfer of sunset yellow and tartrazine, resulted in increased oxidation currents. Ding et al. prepared a nano-hybrid of electrochemically reduced graphene oxide $\left(\mathrm{MnO}_{2}\right.$ NRsERGO) decorated with manganese dioxide nanorods, which was used to modify the glassy carbon electrode (GCE) [51]. Because of the strong catalytic activity of $\mathrm{MnO}_{2} \mathrm{NR}_{\mathrm{S}}$ and the high adsorption capacity and excellent conductivity of ERGO, the modified glassy carbon electrode showed excellent analytical performance such as high sensitivity, high selectivity and low cost. Moreover, it was used to detect sunset yellow (SY) in soft drinks with wide linear range and low detection limit. Zhao et al. developed a molecularly imprinted copolymer sensor (MIP-PMDB/POPD-GCE) by electropolymerizing $\mathrm{m}$-dihydroxybenzene $(\mathrm{m}-\mathrm{db})$ and $\mathrm{o}$ phenylenediamine (o-PD) and then directly fixing the copolymer on the surface of glass carbon electrode (GCE) [32]. Due to the fact that the copolymer is filled with nanopores which can bind tartrazine molecules, tartrazine in soft drinks can be determined quickly.

In addition to glassy carbon electrode, carbon paste electrode, graphene electrode and metal electrode can also be used for the detection of colorants in soft drinks [26,28,31,35,39,52]. For example, Ya et al. simply synthesized a Zinc oxide nanoflower (ZnONF) to modify the carbon paste electrode to obtain a zinc oxide nanoflower modified carbon paste electrode ( $\mathrm{ZnONF} / \mathrm{CPE}$ ), which was used to detect sunset yellow in soft drinks.

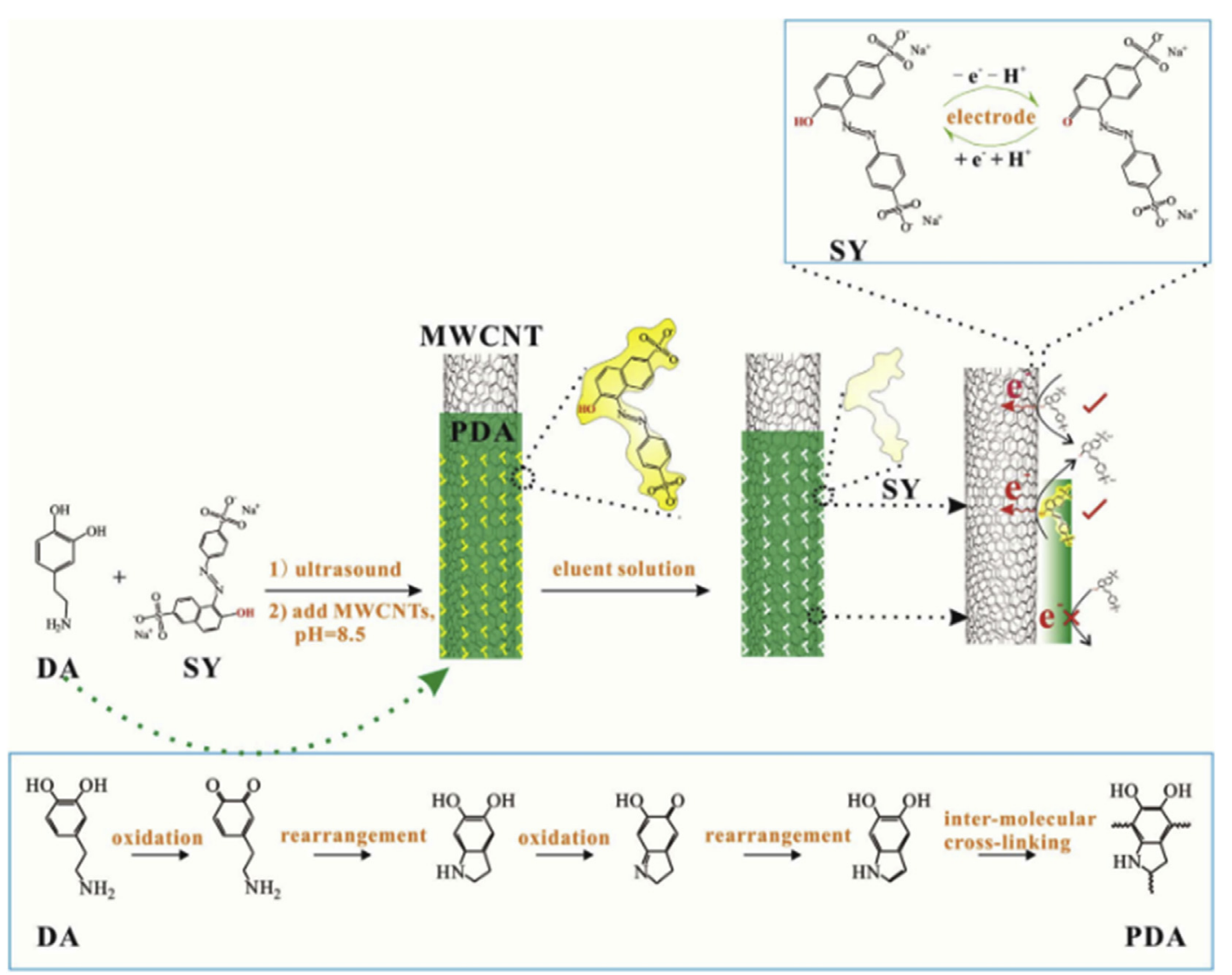

Fig. 1. Schematic preparation of MWCNT@polydopamine (PDA) and sunset yellow (SY) sensing. Reprinted with permission from Ref. [48] with permission from the Elsevier, Copyright 2018. 
Under the best conditions, the detection limit is $0.10 \mu \mathrm{g} / \mathrm{L}$. Due to the large specific surface area and high accumulation efficiency of $\mathrm{ZnONF}$, the electrochemical oxidation of sunset yellow was enhanced by ZnONF modified carbon paste electrode (ZnONF/CPE) [52]. Wu et al. used one-pot hydrothermal to fix the $\mathrm{ZnO} / \mathrm{RGO} / \mathrm{ZnO}$ nanocomposite in situ on the zinc foil, and used the obtained $\mathrm{ZnO} / \mathrm{RGO} / \mathrm{ZnO} @ \mathrm{Zn}$ directly as the electrode material to detect sunset yellow in soft drinks, and It shows an ultrahigh sensitivity of $20.25 \mu \mathrm{A} \mu \mathrm{M}^{-1} \mathrm{~cm}^{-2}$, a low LOD (3 $\left.\mathrm{nM}\right)$, a wide linear detection ranging from 0.01 to $5 \mu \mathrm{M}$ [39]. By casting a double-stranded spiral copper (I) onto the surface of a single-walled carbon nanotube modified screen-printed electrode, Nuñez-Dallos et al. reported the voltammetry of amaranth red and tartrazine in soft drinks [31]. Using this method, amaranth red and tartrazine can be detected at $30 \mathrm{nM}$ and $60 \mathrm{nM}(\mathrm{S} / \mathrm{N}=3)$, respectively. The small relative standard deviation $(3.5 \%, \mathrm{n}=7$, three different electrodes) proves the high accuracy of the proposed method. In addition, the organic molecule $\beta$-cyclodextrin can also be used to modify the electrode to enhance the electrochemical detection in soft drinks due to its good adsorption capacity $[36,53]$. Although the most commonly used method for detecting colorants in soft drinks is to modify the working electrode with nanotechnology, in recent years, some methods other than electrochemical sensing have been reported to better detect colorants in soft drinks [54-57]. Among them, surface enhanced Raman spectroscopy (SERS) as a highly sensitive nanotechnology can also be used to detect colorants in soft drinks. For example, Ou et al. used gold nanorods (Au NRs) as SERS substrates and proposed a system to detect Allure red or sunset yellow in soft drinks [55]. In their work, they found that the aspect ratio of gold nanorods is crucial for SERS signals, and the strongest Raman signal enhancement is achieved by special Au NRs (aspect ratio $=2.4$ or 1.8 ). He et al. chose cysteamine to modify the gold nanoparticle SERS substrate, and then detected the acid pigment with weak surface affinity in soft drinks [56]. Because cysteamine has a positively charged group $-\mathrm{NH}_{3}$, it can electrostatically interact with acidic pigments, and hydrogen carbonate bonds between the calcium carbonate and the pigment molecules, making the pigment molecules closer to the surface of the gold nanoparticles. Experimental results show that the method still has good reproducibility under complex conditions, and the results can be detected very quickly and sensitively.

\subsection{Colorants analysis in other foods}

As food cosmetics, food colorants can also be used for other foods, e.g., meat, cake, and candy. It is noticed that food colorants not only provide attractive color, but also help to preserve water. However, according to food colorants regulations, synthetic colorants are not able to be used in meat and its processed products. In this case, only few natural colorants could be applied in meat processing. For example, Carminic acid (CA) is extensively used as a typical red and natural food colorants for meat products. However, the allowable concentration range of CA in foodstuff is set to be $25-500 \mathrm{mg} / \mathrm{kg}$ by the Standardization Administration of China (GB 2760-2014). Therefore, in order to detect CA, Yuan et al. learned that small-sized layered double hydroxides (LDHs) can increase the intensity of the chemiluminescence (CL) of the Luminol- $\mathrm{H}_{2} \mathrm{O}_{2}$ system by more than 950 times [58]. The enhanced chemiluminescence is due to the synergistic effect of the positively charged layer and the intercalated carbonate. The former attracts luminol dianions and increases their local concentration, while the latter forms carbonates and promotes the formation of emissive 3-aminophthalate anions. Therefore, combined with the negative charge nature of $C A$, the author proposed a sensitive chemiluminescence detection CA method based on the LDH-luminol$\mathrm{H}_{2} \mathrm{O}_{2}$ system (Fig. 2) [59]. The surface adsorption of CA causes the positively charged center of the LDH layer to be occupied, which hinders the approach of the Luminol dianion. Similarly, the reducing power of CA inhibits the formation of free radicals, which reduces the interaction between luminol divalent anions and free radicals. In addition, the UV-vis absorption range of CA overlaps with the chemiluminescent emission of the luminescent 3-aminophthalate anion, resulting in the occurrence of chemiluminescent resonance energy transfer. As a result, the synergy of occupied active sites, reduction of free radicals and CL resonance energy transfer leads to quenching of CL emission. Based on this mechanism, they achieved the detection of CA in dried pork slices within a concentration range of $0.5-10 \mu \mathrm{M}$, with a LOD of $0.03 \mu \mathrm{M}$. They also studied the repeatability of the proposed CL sensor. During 11 repeated measurements of $2 \mu \mathrm{M}$ CA, the calculated relative standard deviation was $2.9 \%$, which proved the accurate CA analysis of the designed system. Indeed, the chemiluminescence spectrophotometry used in this report as a traditional detection method has the advantages of low background noise, fast response and simple instrument $[60,61]$, it has been widely used in combination 


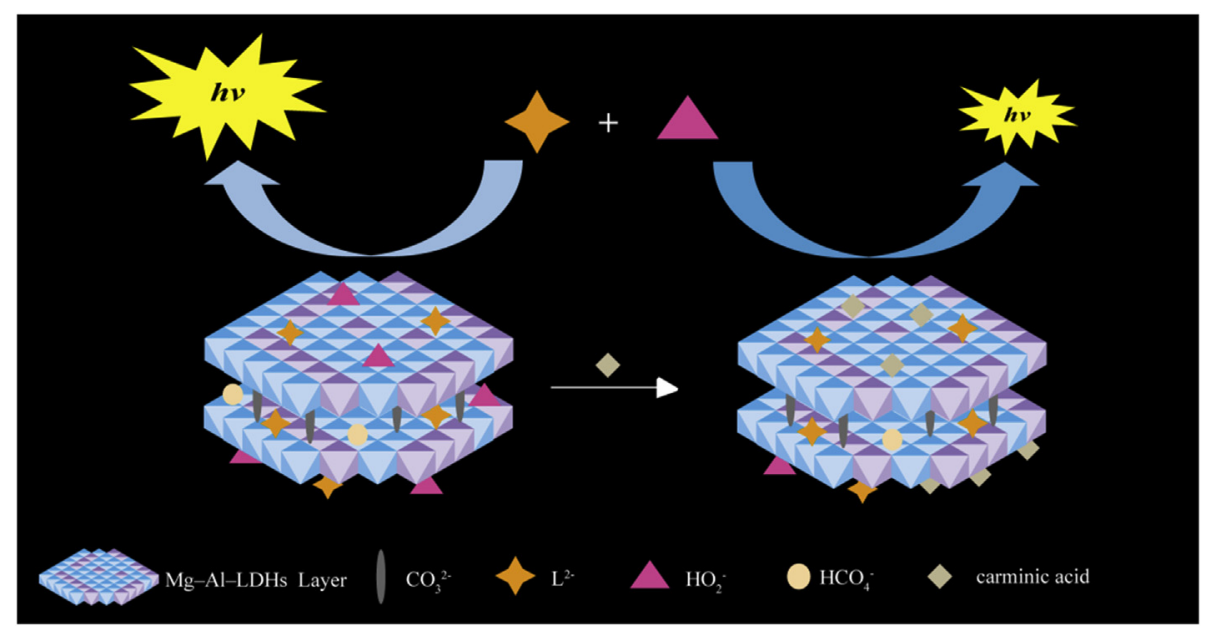

Fig. 2. Schematic representation of the sensing principle of $\mathrm{LDH}$-luminol- $\mathrm{H}_{2} \mathrm{O}_{2}$ system for the detection of CA. Reproduced from Ref. [59] with permission from The American Chemical Society, Copyright 2018.

with the increasingly developed nanotechnology [58,59,62].

Electrochemical methods with high sensitivities have also been applied for the detection of food colorants. For example, Arvand et al. prepared homogeneous bimetallic nickel tin oxide hollow nanospheres by template method to modify carbon paste electrode to obtain a new electrode $(\mathrm{Ni}-\mathrm{Sn}$ oxide NSs/CPE) [34]. The results show that the electrode has higher sensitivity than bare carbon paste electrode in the detection of erythrosine in commercial food such as candy and jelly. This is because the active sites of the reaction are greatly increased due to the high specific surface area of bimetallic hollow nanospheres, and the synergistic action of the two metals can make the electron transfer in $\mathrm{Ni}-\mathrm{Sn}$-oxide NSs easier. However, the specific mechanism of the electrode for the detection of erythrosine in the article needs further study. Zhao et al. used molecular imprinting technology for electrochemical sensing to specifically detect the food pigment erythrosine [33]. In order to improve the sensitivity and stability of the reduced graphene oxide on the surface of the glassy carbon electrode, the author used sodium citrate as a reducing agent and stabilizer, using 2-aminoethanol (2-AET) functionalized, uniformly distributed gold nanoparticles (AuNPs) modified reduced graphene oxide (RGO); in order to improve the selectivity of the sensor, the author used erythrosine as template molecules to obtain molecularly imprinted cavities on the modified electrode surface. Using this method to detect food coloring shows high selectivity and sensitivity. Pogacean et al. prepared the nanocomposites by mixing graphene oxide with $\mathrm{TiO}_{2}-\mathrm{Ag}$ nanoparticles, and then used it to modify the gold electrode to detect amaranth also showed good sensitivity [25]. In addition, chitosan as an easily modified organic molecule can also be used to modify glassy carbon electrodes to better detect food colorants [63].

Although research on how to develop new nanomaterials to modify working electrodes (glass carbon electrodes, carbon paste electrodes, graphene electrodes, metal electrodes, etc.) is currently the most popular direction for the detection of soft drinks and food colorants other than soft drinks, SERS also occupies a proper place in the detection of food colorants due to its fast, sensitive and nondestructive testing characteristics [64,65]. In comparison with ordinary Raman systems, the signals of Raman reporter on the surface of nanoparticles can be greatly enhanced through the surface plasmon resonance-induced electromagnetic enhancement and/or charge transfer mediated chemical enhancement $[66,67]$. To apply SERS technique into the detection of food colorants, the key issue is to obtain a substrate with high SERS activity. Generally speaking, since the random distribution of hot spots on the SERS substrate will cause the SERS signal to show poor repeatability, it is necessary to fabricate nanostructures with uniform gaps. Wu et al. prepared gold nanoparticles with a diameter of $80 \mathrm{~nm}$ into a single layer of uniform gold nanoparticle film by solvent driven self-assembly [68]. Using this film as a SERS substrate, the sensitive analysis of sunset yellow and tartrazine in ppm level was realized. In addition, the author also used it to detect illegal food additives including ciprofloxacin, diethylhexyl phthalate and azodicarbonamide. $\mathrm{Wu}$ et al. used ascorbic acid to reduce silver nitrate, in the presence of a polyvinylpyrrolidone (PVP) 
surfactant, regulates the generation of uniform flower-like silver nanoparticles for SERS substrate analysis to detect carmine in food additives [69]. Using the same method to produce uniform flower-shaped silver nanoparticles, Ai et al. used this silver nanoparticles as a SERS substrate to study four food colorants (food blue, tartrazine, sunset yellow and acid red) [70]. Experimental results show that the LOD of the four colorants is as low as $100 \mathrm{nM}$ (of which tartrazine's LOD is close to $10 \mathrm{nM}$ ). This shows a very high sensitivity for the detection of international standard food coloring. However, not all target analytes can be detected simply by using metal nanoparticles as the substrate, because metal nanoparticles have weak adsorption to some analyte molecules, making it difficult for analyte molecules to access metal nanoparticles [71]. In addition, silver nanoparticles have a tendency to oxidize, so it is difficult to be a long-term SERS substrate [72]. Therefore, when we choose the SERS method to detect food additives or other target analytes, we should take into account the characteristics of the target molecule, and if necessary, modify the nano-substrate to improve its adsorption [73]. In the past four years, most of the relevant reports have used electrochemical methods or SERS. I think this must be inseparable from the word "adsorption". To improve the traditional detection methods with nanotechnology is to make full use of the high specific surface area of nanoparticles to increase the adsorption of target molecules, so as to improve the sensitivity of traditional methods. This is the case with electrode modification, the selection of suitable SERS substrate, even the traditional high performance liquid chromatography (HPLC) [74]. Partial detection methods for food colorants were summarized in Table 1.

\section{Food preservative detection}

Food contains lots of organic compounds, and its time-dependent spoilage is inevitable. Putrescent foods are extremely toxic to Human health and even cause death. Thus, the storage of food is one of the key issues in food industry. Based on the spoilage process, food spoilage primary includes two pathways: contamination by microorganism and oxidation-induced degradation. To extend the food storage period, some chemical compounds are added as ingredients, called as food preservatives [75]. According the function mechanism, these food preservatives can be generally divided two classes, antimicrobial and antioxidant. Antimicrobial preserves food by inhibiting or killing




bacteria/fungus through the denaturation of microbial protein, destruction of cell wall, suppression of enzymatic activity, etc. Many organic and inorganic chemicals can be used as food antimicrobial, including potassium sorbate, sodium benzoate, nitrite, and sulfite, etc [18]. While antioxidant protects food by hindering the oxidation of certain constituents, e.g., fats. Chemicals with good reducing ability are applied as antioxidants, such as butylated hydroxyanisol, butylated hydroxytoluene, tertiary butylhydroquinone, ascorbic acid, polyphenols [75]. Notice that the added food preservatives must be nontoxic, thus the contents need to be strictly limited based on food safety rules. Recent studies indicated that the excess uptake of food preservatives leads to various diseases and even cancer [76-79]. Mohammadzadeh-Aghdash et al. summarized the interaction between food preservatives and serum albumin and revealed that the interaction leads to the activation of inflammatory paths and acceleration of diabetes [80]. Taking this into consideration, the development of sensitive detection methods toward food preservatives is of significance to ensure food safety and Human health. In this section, detection methods on antimicrobial and antioxidant were briefly introduced.

\subsection{Antimicrobial}

Similar to the colorant's detection, electrochemical and optical detection methods are also applicable to food preservatives analysis. And the detection sensitivity and selectivity could be improved with the introduction of nanomaterials. Gold working electrode functionalized with $p$-amino thiophenol and $\mathrm{Au}$ NPs has been applied to the electrochemical detection of nitrite, a typical inorganic food preservative [81]. After processing of electrode, nitrite oxidation on the electrode surface with a characteristic peak potential of $0.76 \mathrm{~V}$ was observed. And the current was found to be proportional to the nitrite concentration. With this system, nitrite detection showed good linearity in concentration range of $0.5-50 \mu \mathrm{g} / \mathrm{mL}$ nitrite and a LOD of $0.12 \mu \mathrm{g} / \mathrm{mL}$. Beyond electrochemical activity, nitrite has some special reactivity toward some oxidant. It is well known that it can react with hydrogen peroxide and produces generate peroxynitrous acid under acidic conditions. The highly reactive peroxynitrous acid can oxidize surface protecting agent, and cause aggregation of nanomaterials. Using this tactic, Yuan et al. reported a fluorescence turn-off method to detect nitrite with hyperbranched polyethyleneimine protected silver nanoclusters (hPEI-Ag NCs) [82]. The sensing was performed under acidic condition in the presence of excess hydrogen peroxide. The surface coating with hyperbranched polyethyleneimine is critical to accomplish the sensing application. That is because the hyperbranched molecular structure endows high stability of Ag NCs toward hydrogen peroxide or sole nitrite. However, the generated peroxynitrous acid destroys the hyperbranched polyethyleneimine and leads to the formation of $\mathrm{Ag} \mathrm{NC}$ aggregates, which subsequently causes the fluorescence quenching (Fig. 3). Based on this system, nitrite detection was achieved with a LOD of $100 \mathrm{nM}$, which is much lower than maximum contamination level in drinking water set by the U.S. Environmental Protection Agency. In addition, this method utilizes a specific reaction, and other anions including $\mathrm{F}^{-}, \mathrm{Cl}^{-}, \mathrm{Br}^{-}, \mathrm{I}^{-}, \mathrm{C}_{6} \mathrm{H}_{5} \mathrm{CO}_{2}^{-}$, $\mathrm{SCN}^{-}, \mathrm{N}_{3}^{-}, \mathrm{IO}_{3}^{-}, \mathrm{IO}_{4}^{-}, \mathrm{BrO}_{3}^{-}, \mathrm{ClO}_{4}^{-}$, citrite $^{3-}, \mathrm{EDTA}^{2-}$,

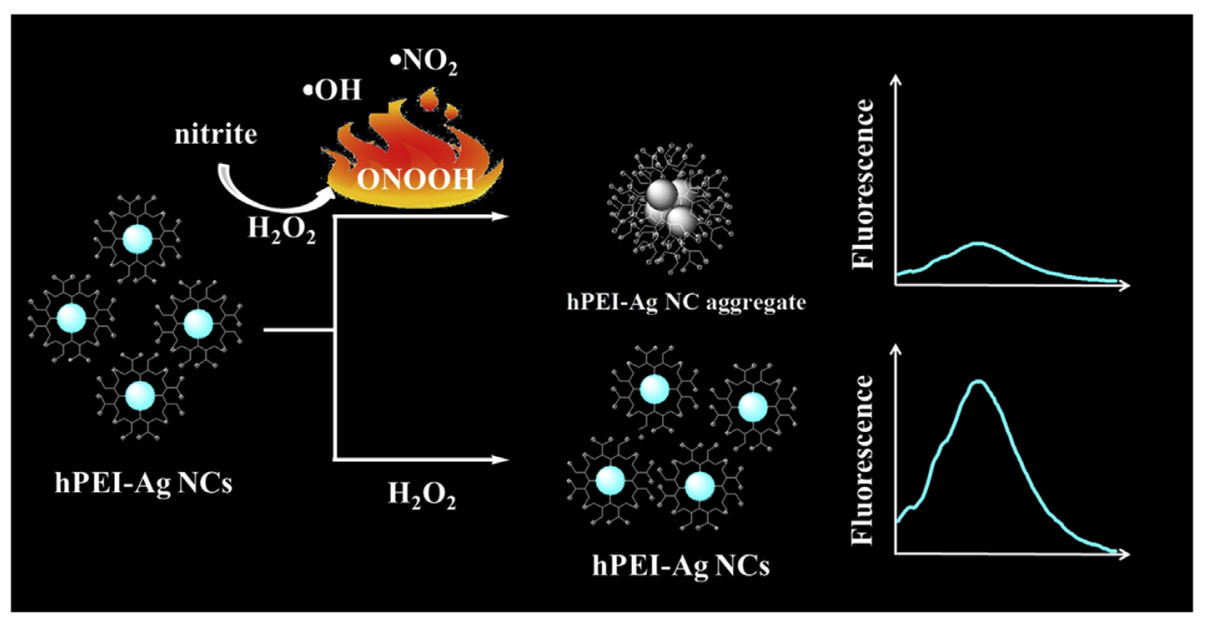

Fig. 3. Schematic illustration of nitrite induced aggregation of hyperbranched polyethyleneimine protected silver nanoclusters (hPEI-Ag NCs) and fluorescence quenching. Reproduced from Ref. [82] with permission from The Royal Society of Chemistry, Copyright 2016. 
$\mathrm{NO}_{3}^{-}, \mathrm{SO}_{3}^{2-}$ and $\mathrm{SO}_{4}^{2-}$, didn't show any visible interference. The good recoveries and small relative standard deviation values proved the practical application of the proposed platform for nitrite analysis in lake water, tap water and seawater samples.

Sulfite has also been widely used as the antimicrobial, which can inhibit the enzymatic activity of some microorganisms. Using methyl green attached $\mathrm{Eu}, \mathrm{Sm}, \mathrm{Mn}$-doped CaS nanoparticles, Wang et al. reported a sensitive sulfite detection platform based on recovery of upconversion luminescence [83]. The sensing mechanism was shown in Fig. 4, methyl green quenched the luminescence of $\mathrm{CaS}$ nanoparticles through fluorescence resonance energy transfer (FRET) mechanism. Introduction of sulfite destroys the molecular structure of methyl green and breaks up FRET process, leading to the enhancement of luminescence. With the use of portable luminescence reader, they achieved sensitive sulfite sensing with a LOD of $2 \mathrm{ng} / \mathrm{mL}$, which is lower than that with commercial test strips $(0.05 \mu \mathrm{g} /$ $\mathrm{mL}$ ). Using nitrobenzofurazan response motif anchored structurally asymmetric naphthorhodamine as the probe; Jin et al. realized fluorimetric sulfite detection in water and wine samples [84]. With the integration of the nucleophilicity of sulfite and the electrostatic attraction mediated reaction kinetics, selective sulfite analysis was achieved with a LOD of $70 \mathrm{nM}$. In addition, this probe was applied for imaging intracellular and endogenous sulfite.

\subsection{Antioxidant}

There are tens to hundreds of antioxidants, which can be used as food preservative. Based on their sources, they can be divided into natural, synthetic and biological antioxidants. Most of them are phenol compounds. Utilizing chemical/electrochemical redox reaction, many works have been reported for the detection of various antioxidants [77,85-89]. For example, using hyperbranched hPEI-Ag NCs as the signal reporters, Yuan et al. achieved tea polyphenol (natural antioxidant) was detected based CL technique [62]. The sensing mechanism is that hydroxyl radicals oxidized Ag $\mathrm{NCs}$ and induce the emission of CL signal (Fig. 5). However, the hydroxyl radicals could be effectively scavenged by tea polyphenols. As a result, the CL intensity decreased. The decreased CL intensity was proportional to the concentration of tea polyphenols. With this method, they realized linear determination of tea polyphenols in the concentration range of $2.52-76.2 \mu \mathrm{M}$. In addition, practical application of the proposed system was verified by detecting tea polyphenols content in three tea samples (green tea, black tea and oolong tea). According to the results, the highest content of tea polyphenols was detected in green tea.

Based on electrochemical strategy, Sandeep et al. also reported the detection of polyphenols (catechol) using graphene nanoribbons/AgNPs/polyphenol oxidase composite modified graphite (Gr) electrode [90]. In this report, polyphenol oxidase (PPO) or catechol oxidase is a copper-containing oxidoreductase enzyme that can catalyze the oxidation of catechol to the corresponding o-quinone. If an enzyme is to be used to modify the electrode, the key is to choose a suitable substrate to fix the enzyme on the electrode without changing the active site of the enzyme. The author chose graphene nanoribbons (GNRs) as the matrix because GNRs have more open-ended structures than graphene and carbon nanotubes, which provide them high density of reaction edges and large active surface area that helps to bind enzyme via electrostatic or $\pi-\pi$ interaction. In addition, because silver nanoparticles are often used as modifiers for biosensors due to their high quantum electron transfer and catalytic properties, and the capping residues on their surface can effectively clamp GNR and PPO enzymes, retaining all the properties of the enzyme, the author chose AgNPs to modify the matrix to further improve the sensitivity and conductivity of GNR. The Gr/GNRs/AgNPs/PPO sensor obtained by modifying the graphite electrode with this method has a lower detection limit $(0.03 \mu \mathrm{M})$ and a wider linear range $(2-2300 \mu \mathrm{M})$ for detecting catechol. Ascorbic acid is also an efficient natural antioxidant, which shows strong reducing ability and can be used in various areas. The detection of ascorbic acid based on optical techniques has also been reported in last few years. Using metal oxides or metal hydroxides as the nanocatalysts, colorimetric sensing of ascorbic acid was achieved [91,92].

In comparison with natural antioxidants, synthetic antioxidants usually show stronger toxicity, making the detection of these synthetic antioxidants important. Up to now, many electrochemical methods toward these synthetic antioxidants have been explored with the combination of molecularly imprinted polymer. Yue et al. reported the rapid detection of tertiary butylhydroquinone using $\mathrm{Pd}-\mathrm{Au}$ bimetallic nanoparticles decorated reduced graphene oxide (rGO) electrode with the surface functionalization of molecularly imprinted polymer [86]. The molecularly imprinted polymer was in-situ generated with tertiary butylhydroquinone as the template molecule. As expected, selective tertiary butylhydroquinone detection was achieved by 




Fig. 4. Schematics of upconversion luminescence (UCL) turn-on sulfite detection using methyl green functionalized upconversion nanoparticles (UCNPs). Reprinted from Ref. [83] with permission from the American Chemical Society, Copyright 2018.

integrating electrochemical analysis. For tertiary butylhydroquinone sensing, the proposed method provided the linear range of $0.5-60 \mu \mathrm{g} / \mathrm{mL}$ with a LOD of $0.046 \mu \mathrm{g} / \mathrm{mL}$. By decorating Au NPs and molecularly imprinted polymer onto the surface of three-dimensional graphite paper electrode, Fan et $a l$. greatly enhanced the detection sensitivity toward tertiary butylhydroquinone [77]. With such a design, tertiary butylhydroquinone was detected in the concentration range of $80 \mathrm{nM}-100 \mu \mathrm{M}$, with a LOD of $12 \mathrm{nM}$. The large surface area of three-dimensional graphite paper plays an important role on the enhanced sensitivity. Organic electrochemical transistor has also been applied for the electrochemical detection of antioxidant. After the modification of gate electrodes with poly(diallyldimethylammoniumchloride) and MWNTs, Xiong et al. reported the electrochemical sensing of gallic acid at very low concentration $(10 \mathrm{nM})$ [93]. This system possesses small operating voltage in comparison with traditional electrochemical



Fig. 5. The sensing mechanism is that hydroxyl radicals oxidized Ag NCs and induce the emission of CL signal. Reproduced from Ref. [62] with permission from The Royal Society of Chemistry, Copyright 2017. methods, and provides a promising tool for portable gallic acid analysis in actual tea beverages.

Due to the advantages of small sample volume, high throughput, and low cost, simultaneous detection of multiple targets in a single run/volume have attracted growing attention. For the electrochemical assays, simultaneous detection of multiple antioxidants is possible with separated voltammetric peaks. As an example, $\mathrm{Ng}$ et al. proposed a fast and sensitive method for the simultaneous detection of tertiary butylhydroquinone, butyl hydroxyanisole and butylated hydroxytoluene [94]. The voltammetric peaks for tertiary butylhydroquinone, butyl hydroxyanisole and butylated hydroxytoluene were observed at $0.278 \mathrm{~V}, 0.503 \mathrm{~V}$, and $0.747 \mathrm{~V}$, respectively. This method allows the detection of tertiary butylhydroquinone, butyl hydroxyanisole and butylated hydroxytoluene at 0.5 , 0.3 , and $0.7 \mu \mathrm{g} / \mathrm{mL}$. And the analysis of these antioxidants in ghee, sunflower oil and salad dressing was realized.

In addition, array-based sensing with the integration of principal component analysis and hierarchical cluster analysis is also capable of multiple targets analysis $[95,96]$. With this strategy, Bordbar et al. developed a colorimetric array for distinguishing 20 various food antioxidants [97]. In this work, $\mathrm{Au}$ NPs and Ag NPs synthesized by six different reducing and/or capping agents (sodium borohydride, chitosan, cetyltrimethylammonium bromide (CTAB), bovine serum albumin (BSA), glucose and polyvinyl pyrrolidone (PVP)) were used as the sensing elements. Interaction between individual antioxidant and these elements causes aggregation or morphological changes of Au NPs and Ag NPs, which generates unique response pattern (Fig. 6). After the acquirement of colorful imaging with a digital camera, the color information was 


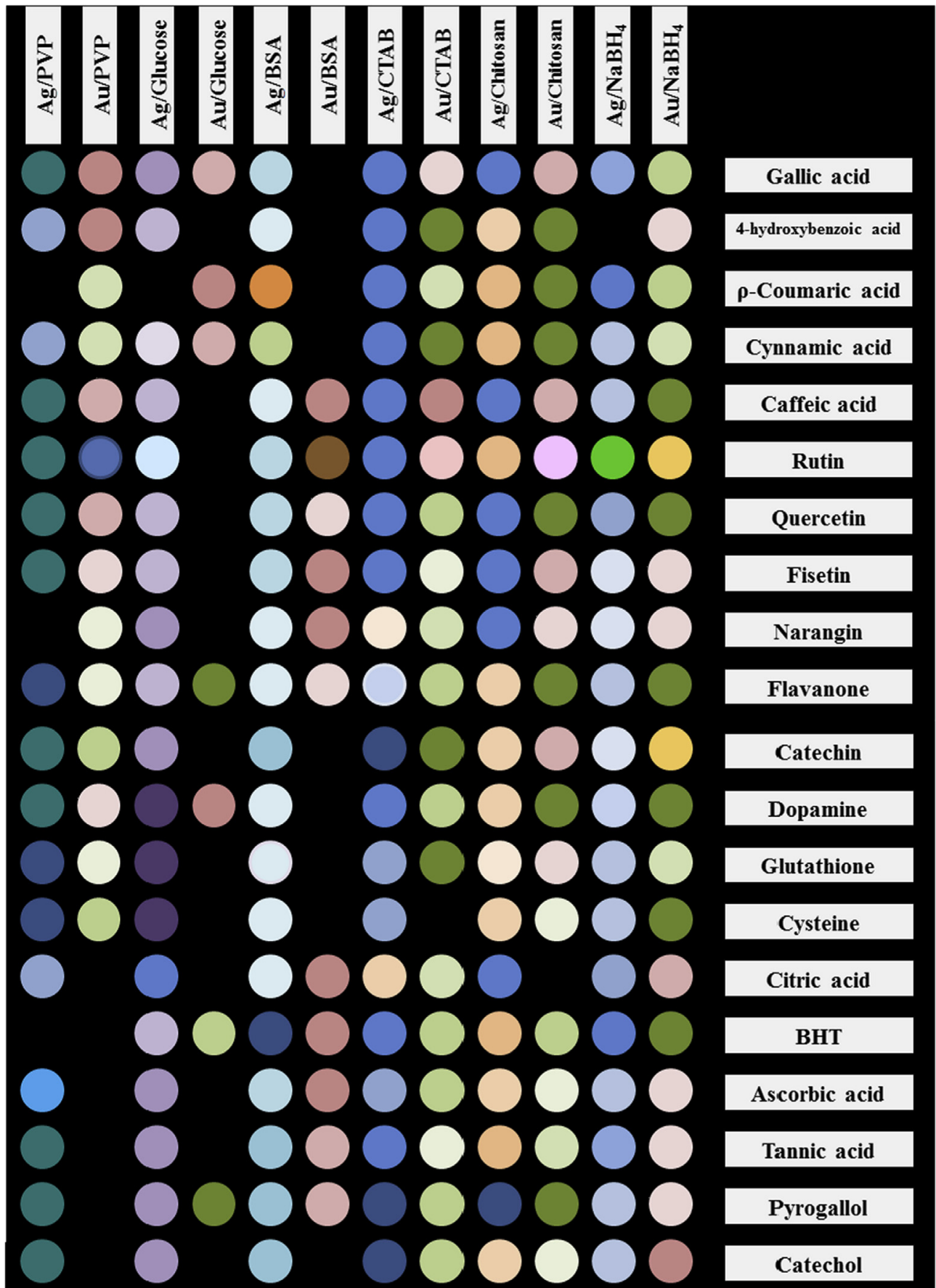

Fig. 6. Color difference maps of 20 types of antioxidant. Reprinted from Ref. [97] with permission from the Springer, Copyright 2018.

analyzed through principal component analysis and hierarchical cluster analysis. All repeated measurements were assigned into the same group, indicating the successful differentiation of these antioxidants. Interestingly, Euclidean Norm was proportional to the antioxidant concentration. Through the calibration, the LODs for gallic acid, caffeic acid, catechin, dopamine, citric acid, butylated hydroxytoluene, and ascorbic acid were determined to be $4.2,13,53,6.9,47,3.5$, and $43 \mathrm{nM}$, respectively. Partial detection methods for food preservatives were summarized in Table 2.

\section{Illegal food additives detection}

In addition to above food additives, some special chemicals are also used to improve the storage or appearance of food. However, these chemicals, 


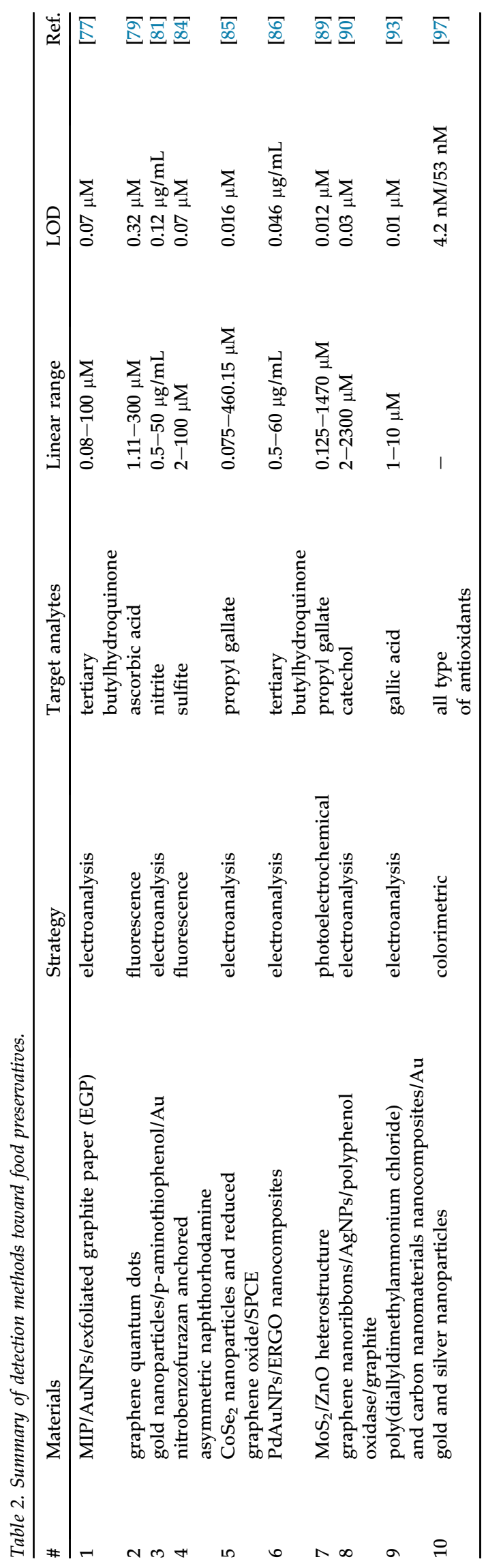

known as illegal food additives, are strictly limited because of their seriously harm to the public health. And most food safety incidents reported in past years are caused by illegally added industrial additives. To control food safety issues, these illegal food additives need to be strictly controlled. Therefore, the development of facile approaches for the detection of these illegal food additives is very important. With the combination of HPLC technique, several works for analyzing illegal food additives have been reported $[98,99]$. In this part, three well-known illegal food additives, including Sudan dye, clenbuterol and melamine, are discussed.

\subsection{Sudan dyes}

Sudan dyes, including Sudan I, Sudan II, Sudan III, Sudan IV, and Sudan black, are a group of oil soluble dyes, which are commonly used in plastics, rubber and paint. As synthetic dye, Sudan dyes possess bright red color and are very stable in comparison with natural colorants. Thus, they have been used for chemical colorant due to advantages of colorfastness and low price. However, Sudan dyes contain azo structure, which is suspected to be carcinogenic to human being. Recently, these Sudan dyes are utilized for food colouration by unscrupulous merchants. In consideration of their harm to Human being, Sudan dye as food additives have been severely prohibited according to the Food Standards Agency.

To detect Sudan dyes, many works based on electrochemical and optical sensing techniques has been reported [100-104]. For example, Ye et al. reported an electrochemical detection platform for the Sudan I sensing by using $\mathrm{CuO}$ nanoparticle-decorated 3D $\mathrm{N}$-doped porous carbon $(\mathrm{CuO} / 3 \mathrm{DNPC})$ modified GCE electrode [102]. The potential of Sudan I become lower upon when approaching electrode, which enhances the current. Using this mechanism, they achieved selective Sudan I detection in a linear concentration range from 2.5 to $100 \mu \mathrm{M}$ with between with a LOD of $0.84 \mu \mathrm{M}$. They also realized the detection of Sudan I in ketchup and chili sauces.

To enhance the detection sensitivity, MahmoudiMoghaddam et al. proposed a screen printed electrochemical sensor by using $\mathrm{La}^{3+}$-doped $\mathrm{Co}_{3} \mathrm{O}_{4}$ nanocubes as electrode substrate [100]. The catalytic character of $\mathrm{La}^{3+}$ greatly decreases the oxidation overpotential of Sudan I and enhances the peak current. With this advantage, Sudan I could be detected in the range of $0.3-300.0 \mu \mathrm{M}$. And the LOD toward Sudan I was calculated to be $0.05 \mu \mathrm{M}$. During the selectivity investigation, metal ions and 
small molecules, including $\mathrm{Ca}^{2+}, \mathrm{Ni}^{2+}, \mathrm{Mn}^{2+}, \mathrm{Pb}^{2+}$, $\mathrm{Mg}^{2+}, \mathrm{Cr}^{2+}, \mathrm{Zn}^{2+}, \mathrm{CN}^{-}, \mathrm{Br}^{-}, \mathrm{K}^{+}, \mathrm{Li}^{+}, \mathrm{Ag}^{+}, \mathrm{SCN}^{-}$, sucrose, fructose, lactose, glucose, and urea, have no interference. In addition, Sudan I detection chili powder, tomato paste, and ketchup sauce have also been achieved. Similarly, using zinc oxide nanoparticles (ZnONPs) as the electrode material, Heydari et al. displayed the electrochemical Sudan III detection by integrating rotatable central composite design and response surface method [101]. With this combination, Sudan III detection was achieved in the linear dynamic ranges of $0.05-1.0$ and 1.0-15.0 $\mu \mathrm{M}$, with a LOD of $2.56 \mathrm{nM}$.

Using Ag NPs as the SERS substrate, Zhao et al. first reported a simple and effective SERS-based method for the detection of Sudan black B [103]. As shown in Fig. 7, they realized the Sudan black B sensing at a concentration of $0.05 \mu \mathrm{g} / \mathrm{mL}$ in standard solutions. Interestingly, $0.1 \mathrm{mg} / \mathrm{kg}$ Sudan black B in black rice extracts was also detected using the proposed SERS-based method. From the perspective of saving resources, reducing costs and reducing environmental pressure, $\mathrm{Hu}$ et al. used tire-derived carbon dots as fluorescent sensors for sensitive detection of Sudan dyes [104]. Because the injection of nitrogen atoms into the carbon dots can lead to a change in the electronic environment and a significant increase in photoluminescence efficiency, the author used ammonium persulfate to oxidize the tire and dope with nitrogen atoms to make the prepared carbon dots have a high quantum yield of $23.8 \%$. Because of the internal filtration effect, the carbon dots obtained after adding Sudan I-IV will have a significant decrease in fluorescence. This method is used to realize the sensitive detection of Sudan I-IV in chilli powder with the LODs are 0.17, $0.21,0.53$ and $0.62 \mu \mathrm{M}$ for Sudan I, II, III and IV, respectively.

\subsection{Clenbuterol}

Clenbuterol is a well-known $\beta_{2}$-adrenergic receptor agonist, which is used for the cure of chronic pulmonary and bronchial diseases. However, because of its capability to increase the lean meat rate and feed conversion rate of animals, clenbuterol is illegally used as a growth promoter and feed additive for livestock and poultry. Unfortunately, excessive clenbuterol residue in meat causes serious health problems, including cardiovascular diseases, central nervous diseases, and even acute poisoning. As a result, the monitoring of clenbuterol residue in meat samples is important to Human health. It is thus important to develop sensitive and selective method for clenbuterol sensing.
Toward this goal, a few systems have been explored based on electrochemical and optical response [105-109]. For instance, Zhang et al. reported an electrochemical sensor for clenbuterol detection by using polyoxometalate and zirconium dioxide nanocomposites as electrode material [106]. The used electrode material negatively shifts the oxidation potentials of clenbuterol and enhances the peak current. With this design, they achieved linear clenbuterol detection in the concentration range from 0.1 to $1000 \mu \mathrm{M}$, with a LOD of $5.03 \mathrm{nM}$. Using pork as the real sample, they realized clenbuterol with good recoveries (94.4-102.3\%).

Beyond electrochemical sensing, optical analysis of clenbuterol has also been reported. Sun et al. proposed a SERS-based approach to detect clenbuterol by using GO/Au NPs as the SERS substrate [107]. Through controlling the depositing density of Au NPs, highly dense hot spots with strong electromagnetic coupling effect was produced. Using $\mathrm{GO} / \mathrm{Au}$ NPs as the substrate, Raman signal of clenbuterol was enhanced 4.8 times in comparison with the use of sole Au NPs substrate. This proposed SERS-based method allows the selective clenbuterol detection with a LOD of $33.4 \mathrm{nM}$. Also based on gold nanoparticles, Muthaiah Shellaiah et al. developed a nanodiamond conjugated gold nanoparticles (ND@AuNPs) as colorimetric probe for the detection of clenbuterol in urine [108]. Through modifying nanodiamonds to have thiol groups, and then reacting with gold nanoparticles to obtain highly stable conjugates (ND@AuNPs). The complexation of the amide $(-\mathrm{C}=\mathrm{O}$ and $-\mathrm{N}-\mathrm{H})$ functional groups of ND@AuNPs with clenbuterol causes aggregation and changes the color from wine red to purple. This colorimetric method is used for selective detection of clenbuterol with a LOD of $0.49 \mathrm{nM}$.

To further improve the selectivity, immunofluorescence method for clenbuterol analysis has been developed. Conventional immunofluorescence technology requires complicated labeling procedure, which diminishes the sensing performances. To avoid labeling, Yao et al. proposed a competition strategy using $\mathrm{S} / \mathrm{N}$-doped carbon quantum dots and clenbuterol antibody [105]. In their work, clenbuterol antibody quenches the fluorescence of carbon quantum dots via amine-amine amine-amine coupling reaction. The introduction clenbuterol leads to the formation of stable clenbuterol antibody-clenbuterol complex, which liberates carbon quantum dots and enhances the fluorescence. This approach shows fluorimetric clenbuterol sensing in the concentration range from 0.07 to $1.7 \mathrm{ng} / \mathrm{mL}$, with a LOD of $23 \mathrm{pg} / \mathrm{mL}$. In addition, 


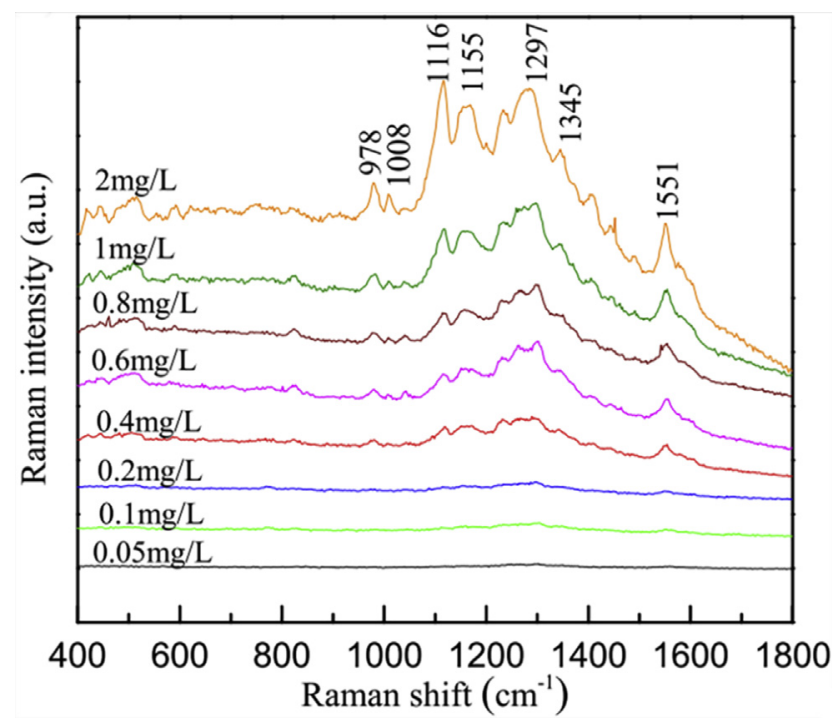

Fig. 7. Representative SERS spectra of Sudan black B standard solutions with different concentration. Reprinted from Ref. [103] with permission from the Elsevier, Copyright 2019.

nanoparticles and gold nanoclusters can also be used in immunofluorescence technology. For example, Peng et al. proposed a fluorescence immunoassay method combined with immunomagnetic separation [109]. In order to reduce the matrix effect, the author added immunomagnetic nanoparticles to the swine urine sample to separate the target substance immediately and effectively。 Because it is loaded with Bovine serum albumingold nanoclusters (BSA-AuNCs) and artificial antigens, nanoflowers have the dual functions of fluorescence signal output and biological recognition. After adding this fluorescent-hybrid nanoflowers to the sample solution that has been magnetically separated, the fluorescence intensity of the supernatant obtained by magnetic separation is directly proportional to the concentration of clenbuterol. The assay has a linear response in the $0.5 \mathrm{ng} / \mathrm{mL}$ to $40 \mathrm{ng} / \mathrm{mL}$ clenbuterol concentration range, and $0.167 \mathrm{ng} / \mathrm{mL}$ limit of detection.

\subsection{Melamine}

Melamine, a triazine nitrogen-containing heterocyclic organic compound, has high nitrogen content of $66.67 \%$. Since normal technique for evaluating the protein content in food is based on Kjeldahl method, in order to increase the tested protein content, this chemical has been added to milk by some unscrupulous merchants. However, excessive uptake of melamine induces serious health problems, such as kidney disease, chronic nephritis and bladder cancer. Then, the exploration of rapid, sensitive, and accurate method for detecting melamine in food, especially in milk is essential to Human health.

In view of its amino-contained structure, melamine shows high binding affinity to metal, like gold and silver. And thus, much effort has been dedicated to develop metal nanoparticle-based system for melamine detection [110-114]. For example, Ren et al. developed a competitive melamine immunosensor based on branched polyethyleneimine functionalized $\mathrm{rGO}$ and $\mathrm{Au}$ NPs modified electrode [112]. In this report, the author first assembled the negatively charged sodium 3mercaptopropane sulfonate to the $\mathrm{Au}$ electrode through the $\mathrm{Au}-\mathrm{S}$ bond. On this basis, the positively charged branched polyethyleneimine functionalized reduced graphene oxide (BPEIGn) (prepared by a one-step catalytic reaction) is absorbed on the electrode surface through electrostatic interaction. Then, because the surface of BPEIGn is filled with many amino groups and positive charges, the negatively charged AuNPs are fixed on the electrode through covalent interaction and electrostatic adsorption. Subsequently, melamine is attached to the electrode surface by forming hydrogen bonds with polyethyleneimine. The flexible structure of branched polyethyleneimine and its abundant amino groups can enhance its interaction with melamine. Finally, using bovine serum albumin (BSA) to block non-specific absorption sites to obtain a melamine immunosensor modified electrode. It is precisely because of the principle of specific binding between antigen and antibody and the double amplification of BPEIGn and AuNPs that this sensor has excellent specificity and sensitivity. Therefore, using $\mathrm{K}_{3}\left[\left(\mathrm{Fe}(\mathrm{CN})_{6}\right] / \mathrm{K}_{4}[\mathrm{Fe}\right.$ $\left.(\mathrm{CN})_{6}\right]$ solution as the working electrolyte, they achieved melamine analysis in the concentration range of $1 \times 10^{-6}$ to $1 \mu \mathrm{M}$, with a LOD of $2.66 \times 10^{-7} \mu \mathrm{M}$.

Nie et al. proposed a bowl-like pore array that consists of hollow Au/Ag alloy nanoparticles, acting as hot-spots [111]. The designed bowl-like pore array showed high SERS activity toward melamine. Through the finite-difference time-domain simulations, the SERS enhancement mechanism was attributed to the surface plasmon resonance of alloy nanoparticles. The Raman shift $681 \mathrm{~cm}^{-1}$ that originates from the in-plane deformation modes of triazine ring was used as the reporting signal. The intensity of signal increased upon increasing melamine concentration from $1 \mathrm{nM}$ to $10 \mu \mathrm{M}$. To prove the practical application of this method, milk samples spiked with melamine was investigated. According to the results, melamine in milk could be detected as low as $0.1 \mathrm{ppm}$, which is 10 times lower 


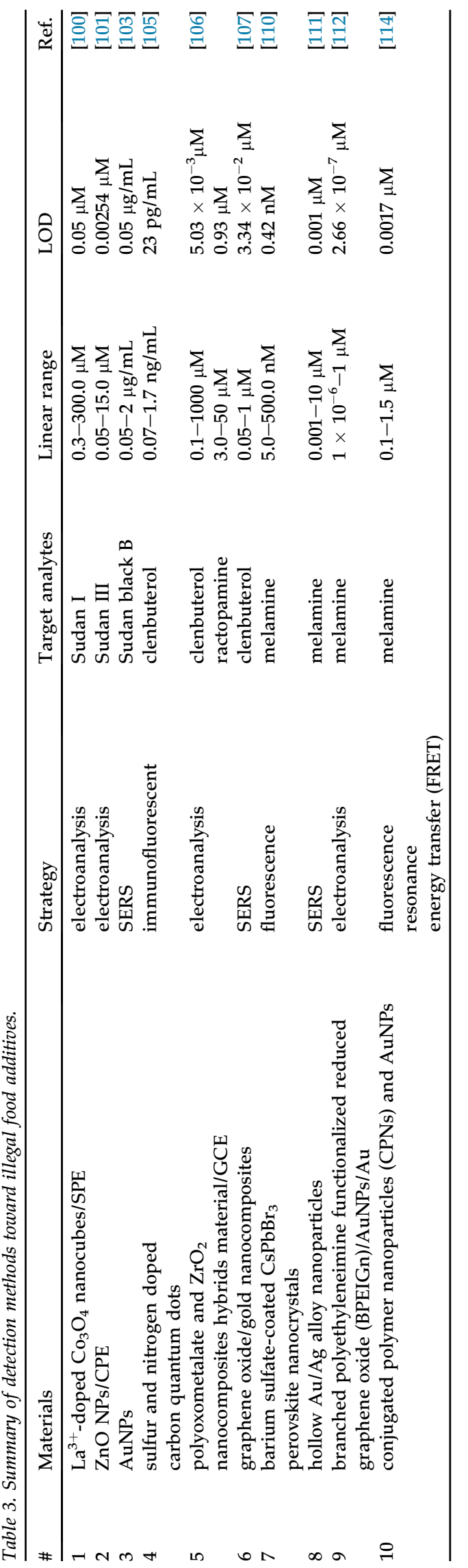

than the permission set by Food and Drug Administration (FDA).

Beyond electrochemical reactivity and SERS activity, Au NPs are also good fluorescence quenchers to organic and inorganic fluorophores. Using barium sulfate-coated $\mathrm{CsPbBr}_{3}$ perovskite nanocrystals $\left(\mathrm{CsPbBr}_{3} \mathrm{NCs}_{\mathrm{B}} @ \mathrm{BaSO}_{4}\right)$ as the fluorophore and $\mathrm{Au}$ NPs as the quencher, $\mathrm{Li}$ et al. reported a fluorescence turn-on method for the detection of melamine [110]. The approaching of Au NPs quenched the fluorescence from $\mathrm{CsPbBr}_{3}$ $\mathrm{NCs} @ \mathrm{BaSO}_{4}$ because of the intense electromagnetic local field around Au NP surface. Addition of melamine weakened the binding affinity between $\mathrm{Au}$

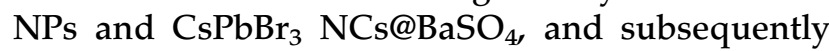
enhanced the fluorescence. With use of this strategy, they realized sensitive melamine determination with a LOD of $0.42 \mathrm{nM}$. In addition, this method provided good stability toward melamine sensing with small relative standard deviation of $4.0 \%$, and could be applied to the analysis of dairy product samples. Partial detection methods for illegal food additives were summarized in Table 3.

\section{Conclusion and perspectives}

In this review, we have displayed an overview on the recent progresses of nanomaterial-assisted food additives analysis based on optical and electrochemical techniques. The unique chemical and electrochemical properties of nanomaterial endow the sensitive detection of food additives including colorants, preservatives and illegal additives in food samples. These sensing systems usually show LODs at $\mathrm{nM}$ or $\mu \mathrm{M}$ levels, which meet the permission of expected food additives set by FDA or EPA. With the combination of multiplex detection tactic and array-based sensing, a few examples accomplish the multiple targets analysis.

Although the present works achieve successful analysis of various food additives using nanomaterial-based optical and/or electrochemical systems, there are still some challenges need to be solved. First, the highly reactive surface of nanomaterials limits their use in complicated conditions. That is, some chemicals or substrates may have interference to the sensing of food additives. However, food extracts usually contain many compounds, including targets and potential interferents. To avoid the false results, an extra separation process might be helpful before analysis. Second, the design of food additives detection system with a universal strategy is lacking. For most systems, the sensing mechanisms are applicable for specific targets. Other food additives with diverse molecular 
structures can not to be detected with similar systems. Thus, the exploration of a simple strategy for constructing versatile detection systems is appealing. In consideration of the molecular recognition nature, molecularly imprinted polymer technique may provide solution for this issue. By using different molecular templates, specific detection of various food additives with similar protocols is possible. Third, sole food additive is generally detected based on nanomaterial-assisted analysis. It is indicated in many works that food process is usually associated with different food additives. For example, colorants and preservatives exist at the same time. To further evaluate the food safety, the detection of multiple food additives from food samples needs to be finished in a single run/test. As mentioned above, electrochemical methods possess possibility to detect several food additives simultaneously through different voltammetric peaks. The overlap of adjacent voltammetric peaks may have negative effect. To realize multiple food additive analysis, array-based sensing might be a promising tool. There are several advantages to array-based sensing, including no demand of specific interaction, high-through, and wide signal input. In this case, to boost the analysis of multiple food additives, array-based detection with the combination of nanomaterials is worthy to be tried. With the assistance of microchip technology, portable analysis of multiple food additive with sensor array is also possible in near future.

\section{Conflicts of interest}

The authors declare no competing financial interest.

\section{Acknowledgment}

This work was supported by the Beijing Natural Science Foundation (2202038), and the National Natural Foundation of China (22074005 and 21605003).

\section{References}

[1] Blekas GA. Food additives: classification, uses and regulation. In: Caballero B, Finglas PM, Toldrá F, editors. Encyclopedia of food and health. Oxford: Academic Press; 2016. p. 731-6.

[2] Cooper PL, Marshall MR, Gregory III JF, Otwell WS. Ion chromatography for determining residual sulfite on shrimp. J Food Sci 1986;51:924-8.

[3] Ferrer Amate C, Unterluggauer H, Fischer RJ, FernandezAlba AR, Masselter S. Development and validation of a LCMS/MS method for the simultaneous determination of aflatoxins, dyes and pesticides in spices. Anal Bioanal Chem 2010;397:93-107.
[4] Lakshmi UR, Srivastava VC, Mall ID, Lataye DH. Rice husk ash as an effective adsorbent: evaluation of adsorptive characteristics for Indigo Carmine dye. J Environ Manag 2009:90:710-20.

[5] Ma Y, Zhang G, Pan J. Spectroscopic studies of DNA interactions with food colorant indigo carmine with the use of ethidium bromide as a fluorescence probe. J Agric Food Chem 2012;60:10867-75.

[6] Carlos KS, Treblin M, de Jager LS. Comparison and optimization of three commercial methods with an LC-MS/MS method for the determination of sulfites in food and beverages. Food Chem 2019;286:537-40.

[7] Jia W, Ling Y, Lin Y, Chang J, Chu X. Analysis of additives in dairy products by liquid chromatography coupled to quadrupole-orbitrap mass spectrometry. J Chromatogr A 2014;1336:67-75.

[8] Kaufmann A, Widmer M, Maden K, Butcher P, Walker S. Analysis of a variety of inorganic and organic additives in food products by ion-pairing liquid chromatography coupled to high-resolution mass spectrometry. Anal Bioanal Chem 2018;410:5629-40.

[9] Hao P-P, Ni J-R, Sun W-L, Huang W. Determination of tertiary butylhydroquinone in edible vegetable oil by liquid chromatography/ion trap mass spectrometry. Food Chem 2007;105:1732-7.

[10] Herzog Gregoire, Kam Victor, Berduque Alfonso, Arrigan Damien WM. Detection of food additives by voltammetry at the liquid-liquid interface. J Agric Food Chem 2008;56:4304-10.

[11] Majid A, Mehrnoosh S, Masoomeh Sayyar A, Asadollah M. Mediated electrochemical method for the determination of indigo carmine levels in food products. Talanta 2017;173: 60-8.

[12] Medeiros RA, Lourencao BC, Rocha-Filho RC, FatibelloFilho O. Flow injection simultaneous determination of synthetic colorants in food using multiple pulse amperometric detection with a boron-doped diamond electrode. Talanta 2012;99:883-9.

[13] Harp BP, Miranda-Bermudez E, Barrows JN. Determination of seven certified color additives in food products using liquid chromatography. J Agric Food Chem 2013;61: 3726-36.

[14] Misra R, Acharya S, Sahoo SK. Cancer nanotechnology: application of nanotechnology in cancer therapy. Drug Discov Today 2010;15:842-50.

[15] Cai J, Niu H, Li Z, Du Y, Cizek P, Xie Z, et al. High-performance supercapacitor electrode materials from cellulose-derived carbon nanofibers. ACS Appl Mater Interfaces 2015;7:14946-53.

[16] Liu Y, Kumar S. Polymer/carbon nanotube nano composite fibers-a review. ACS Appl Mater Interfaces 2014;6:6069-87.

[17] Chi D, Sun D, Yang Z, Xing Z, Li Z, Zhu Q, et al. Bifunctional nest-like self-floating microreactor for enhanced photothermal catalysis and biocatalysis. Environ Sci Nano 2019;6:3551-9.

[18] Pathakoti K, Manubolu M, Hwang HM. Nanostructures: current uses and future applications in food science. J Food Drug Anal 2017;25:245-53.

[19] Bajpai VK, Kamle M, Shukla S, Mahato DK, Chandra P, Hwang SK, et al. Prospects of using nanotechnology for food preservation, safety, and security. J Food Drug Anal 2018;26:1201-14.

[20] Gupta A, Eral HB, Hatton TA, Doyle PS. Nanoemulsions: formation, properties and applications. Soft Matter 2016;12: 2826-41.

[21] Correa DS, Pavinatto A, Mercante LA, Mattoso LHC, Oliveira JE, Riul A. Chemical sensors based on hybrid nanomaterials for food analysis. Nanobiosensors 2017: 205-44.

[22] Wang Y, Duncan TV. Nanoscale sensors for assuring the safety of food products. Curr Opin Biotechnol 2017;44: $74-86$. 
[23] Lv M, Liu Y, Geng J, Kou X, Xin Z, Yang D. Engineering nanomaterials-based biosensors for food safety detection. Biosens Bioelectron 2018;106:122-8.

[24] Downham A, Collins P. Colouring our foods in the last and next millennium. Int J Food Sci Technol 2000;35:5-22.

[25] Pogacean F, Rosu M-C, Coros M, Magerusan L, Moldovan M, Sarosi C, et al. Graphene/TiO2-Ag based composites used as sensitive electrode materials for amaranth electrochemical detection and degradation. J Electrochem Soc 2018;165:B3054-9.

[26] Gao Y, Wang L, Zhang Y, Zou L, Li G, Ye B. Electrochemical behavior of amaranth and its sensitive determination based on Pd-doped polyelectrolyte functionalized graphene modified electrode. Talanta 2017;168:146-51.

[27] Wang M, Cui M, Zhao M, Cao H. Sensitive determination of Amaranth in foods using graphene nanomeshes. J Electrochem Soc 2018;809:117-24.

[28] Karim-Nezhad G, Khorablou Z, Zamani M, Seyed Dorraji P, Alamgholiloo M. Voltammetric sensor for tartrazine determination in soft drinks using poly ( $\mathrm{p}$-aminobenzenesulfonic acid)/zinc oxide nanoparticles in carbon paste electrode. J Food Drug Anal 2017;25:293-301.

[29] Sierra-Rosales P, Toledo-Neira C, Squella J. Electrochemical determination of food colorants in soft drinks using MWCNT-modified GCEs. Sens Actuators, B 2017;240: 1257-64.

[30] Dorraji PS, Jalali F. Electrochemical fabrication of a novel $\mathrm{ZnO} /$ cysteic acid nanocomposite modified electrode and its application to simultaneous determination of sunset yellow and tartrazine. Food Chem 2017;227:73-7.

[31] Nuñez-Dallos N, Macías MA, García-Beltrán $O$, Calderón JA, Nagles E, Hurtado J. Voltammetric determination of amaranth and tartrazine with a new doublestranded copper (I) helicate-single-walled carbon nanotube modified screen printed electrode. J Electroanal Chem 2018; 822:95-104.

[32] Zhao X, Liu Y, Zuo J, Zhang J, Zhu L, Zhang J. Rapid and sensitive determination of tartrazine using a molecularly imprinted copolymer modified carbon electrode (MIPPmDB/PoPD-GCE). J Electroanal Chem 2017;785:90-5.

[33] Zhao X, Hu W, Wang Y, Zhu L, Yang L, Sha Z, et al. Decoration of graphene with 2-aminoethanethiol functionalized gold nanoparticles for molecular imprinted sensing of erythrosine. Carbon 2018;127:618-26.

[34] Arvand M, Pourhabib A, Asadi M. Template-based synthesis of uniform bimetallic nickel-tin oxide hollow nanospheres as a new sensing platform for detection of erythrosine in food products. Sens Actuators, B 2018;255: 1716-25.

[35] Zhao X, Ding J, Bai W, Wang Y, Yan Y, Cheng Y, et al. PEDOT: PSS/AuNPs/CA modified screen-printed carbon based disposable electrochemical sensor for sensitive and selective determination of carmine. J Electroanal Chem 2018;824:14-21.

[36] Ma X, Xie Y, Yu Y, Lu L, Liu G, Yu Y, et al. Three-dimensional $\beta$-cyclodextrin functionalized graphene aerogels: an enhanced electrochemical sensing platform for quantification of Ponceau 4R. J Electroanal Chem 2018;823:437-44.

[37] Hobbs CA, Koyanagi M, Swartz C, Davis J, Maronpot R, Recio L, et al. Genotoxicity evaluation of the naturallyderived food colorant, gardenia blue, and its precursor, genipin. Food Chem Toxicol 2018;118:695-708.

[38] Ji W, Zhang $M$, Wang T, Wang X, Zheng Z, Gong J. Molecularly imprinted solid-phase extraction method based on SH-Au modified silica gel for the detection of six Sudan dyes in chili powder samples. Talanta 2017;165: $18-26$.

[39] Wu X, Zhang X, Zhao C, Qian X. One-pot hydrothermal synthesis of $\mathrm{ZnO} / \mathrm{RGO} / \mathrm{ZnO} @ \mathrm{Zn}$ sensor for sunset yellow in soft drinks. Talanta 2018;179:836-44.

[40] Bakheet AAA, Zhu XS. Poly(ionic liquid) immobilized magnetic nanoparticles as sorbent coupled with fluorescence spectrophotometry for separation/analysis of Allura red. J Mol Liq 2017;242:900-6.

[41] Tiwari S, Deb MK. Modified silver nanoparticles-enhanced single drop microextraction of tartrazine in food samples coupled with diffuse reflectance Fourier transform infrared spectroscopic analysis. Anal Methods 2019;11:3552-62.

[42] Díaz de León Zavala E, Torres Rodríguez LM, MontesRojas A, Torres Mendoza VH, Liñán González AE. Study of electrochemical determination of capsaicin and dihydrocapsaicin at carbon paste electrodes modified by $\beta$ cyclodextrin. J Electroanal Chem 2018;814:174-83.

[43] Hassan AHA, Sappia L, Moura SL, Ali FHM, Moselhy WA, Sotomayor M, et al. Biomimetic magnetic sensor for electrochemical determination of scombrotoxin in fish. Talanta 2019;194:997-1004.

[44] Wang H, Yao S, Liu Y, Wei S, Su J, Hu G. Molecularly imprinted electrochemical sensor based on $\mathrm{Au}$ nanoparticles in carboxylated multi-walled carbon nanotubes for sensitive determination of olaquindox in food and feedstuffs. Biosens Bioelectron 2017;87:417-21.

[45] Kokulnathan T, Chen SM. Robust and selective electrochemical detection of antibiotic residues: the case of integrated lutetium vanadate/graphene sheets architectures. J Hazard Mater 2020;384:121304.

[46] Gan T, Sun J, Meng W, Song L, Zhang Y. Electrochemical sensor based on graphene and mesoporous $\mathrm{TiO}_{2}$ for the simultaneous determination of trace colourants in food. Food Chem 2013;141:3731-7.

[47] Arvand M, Zamani M, Ardaki MS. Rapid electrochemical synthesis of molecularly imprinted polymers on functionalized multi-walled carbon nanotubes for selective recognition of sunset yellow in food samples. Sens Actuators, B 2017;243:927-39.

[48] Yin ZZ, Cheng SW, Xu LB, Liu HY, Huang K, Li L, et al. Highly sensitive and selective sensor for sunset yellow based on molecularly imprinted polydopamine-coated multi-walled carbon nanotubes. Biosens Bioelectron 2018; 100:565-70.

[49] Rovina K, Siddiquee S, Shaarani SM. Highly sensitive electrochemical determination of sunset yellow in commercial food products based on CHIT/GO/MWCNTs/ AuNPs/GCE. Food Contr 2017;82:66-73.

[50] Li L, Zheng H, Guo L, Qu L, Yu L. Construction of novel electrochemical sensors based on bimetallic nanoparticle functionalized graphene for determination of sunset yellow in soft drink. J Electroanal Chem 2019;833:393-400.

[51] Ding Z, Deng P, Wu Y, Tian Y, Li G, Liu J, et al. A novel modified electrode for detection of the food colorant sunset yellow based on nanohybrid of $\mathrm{MnO}_{2}$ nanorods-decorated electrochemically reduced graphene oxide. Molecules 2019; 24:1178.

[52] Ya Y, Jiang C, Li T, Liao J, Fan Y, Wei Y, et al. A zinc oxide nanoflower-based electrochemical sensor for trace detection of sunset yellow. Sensors 2017;17:545.

[53] Ma X, Chen D, Tu X, Gao F, Xie Y, Dai R, et al. Ratiometric electrochemical sensor for sensitive detection of sunset yellow based on three-dimensional polyethyleneimine functionalized reduced graphene oxide aerogels@Au nanoparticles/SH- $\beta$-cyclodextrin. Nanotechnology 2019;30: 475503.

[54] Yang H, Long Y, Li H, Pan S, Liu H, Yang J, et al. Carbon dots synthesized by hydrothermal process via sodium citrate and $\mathrm{NH}_{4} \mathrm{HCO}_{3}$ for sensitive detection of temperature and sunset yellow. J Colloid Interface Sci 2018;516: 192-201.

[55] Ou Y, Wang X, Lai K, Huang Y, Rasco BA, Fan Y. Gold nanorods as surface-enhanced Raman spectroscopy substrates for rapid and sensitive analysis of allura red and sunset yellow in beverages. J Agric Food Chem 2018;66: 2954-61.

[56] He H, Li P, Tang X, Lin D, Xie A, Shen Y, et al. Developing cysteamine-modified SERS substrate for detection of acidic 
pigment with weak surface affinity. Spectrochim Acta Mol Biomol Spectrosc 2019;212:293-9.

[57] Neng J, Xu K, Wang Y, Jia K, Zhang Q, Sun P. Sensitive and selective detection of new red colorant based on surfaceenhanced Raman spectroscopy using molecularly imprinted hydrogels. Appl Sci 2019;9:2672.

[58] Feng P, Yilin Z, Zhiqin Y, Chao L. Determination of alizarin red $S$ based on layered double hydroxides-improved chemiluminescence from hydrogen peroxide and luminol. Anal Methods 2017;9:6468-73.

[59] Pan F, Zhang Y, Yuan Z, Lu C. Sensitive and selective carmine acid detection based on chemiluminescence quenching of layer doubled hydroxide-luminol- $\mathrm{H}_{2} \mathrm{O}_{2}$ system. ACS Omega 2018;3:18836-42.

[60] Su M, Chen P, Sun H. Development and analytical application of chemiluminescence with some super normal metal complexes as oxidant. Trac Trends Anal Chem 2018; 100:36-52.

[61] Siraj N, El-Zahab B, Hamdan S, Karam TE, Haber LH, Li M, et al. Fluorescence, phosphorescence, and chemiluminescence. Anal Chem 2016;88:170-202.

[62] Zhao S, Chen C, Li Z, Yuan Z, Lu C. Hydroxyl radical induced chemiluminescence of hyperbranched polyethyleneimine protected silver nanoclusters and its application in tea polyphenols detection. Anal Methods 2017;9: 3114-20.

[63] Magerusan L, Pogacean F, Coros M, Socaci C, Pruneanu S, Leostean C, et al. Green methodology for the preparation of chitosan/graphene nanomaterial through electrochemical exfoliation and its applicability in Sunset Yellow detection. Electrochim Acta 2018;283:578-89.

[64] Joshua CG, Tonya X, Siyue G, Yanqi Q, Lili H. Rapid identification of artificial and natural food colorants with surface enhanced Raman spectroscopy. Food Contr 2018;92: 267-75.

[65] Qin M, Li P, Zhou X, Zhu J, Wang H, Yang L. Cationic surfactant regulated synthesis of $\mathrm{Au}$ nanorods for sensitive detection of negatively charged colorants by surfaceenhanced Raman spectroscopy. J Raman Spectrosc 2019;50: 809-17.

[66] Andreou C, Neuschmelting V, Tschaharganeh DF, Huang $\mathrm{CH}$, Oseledchyk A, Iacono $\mathrm{P}$, et al. Imaging of liver tumors using surface-enhanced Raman scattering nanoparticles. ACS Nano 2016;10:5015-26.

[67] Sharma B, Frontiera RR, Henry A-I, Ringe E, Van Duyne RP. SERS: materials, applications, and the future. Mater Today 2012;15:16-25.

[68] Wu Y, Yu W, Yang B, Li P. Self-assembled two-dimensional gold nanoparticle film for sensitive nontargeted analysis of food additives with surface-enhanced Raman spectroscopy. Analyst 2018;143:2363-8.

[69] Wu YX, Liang P, Dong QM, Bai Y, Yu Z, Huang J, et al. Design of a silver nanoparticle for sensitive surface enhanced Raman spectroscopy detection of carmine dye. Food Chem 2017;237:974-80.

[70] Ai YJ, Liang P, Wu YX, Dong QM, Li JB, Bai Y, et al. Rapid qualitative and quantitative determination of food colorants by both Raman spectra and surface-enhanced Raman scattering (SERS). Food Chem 2018;241:427-33.

[71] Jiang T, Wang X, Tang S, Zhou J, Gu C, Tang J. Seedmediated synthesis and SERS performance of graphene oxide-wrapped Ag nanomushroom. Sci Rep 2017;7:9795.

[72] Sharma S, Prakash V, Mehta SK. Graphene/silver nanocomposites-potential electron mediators for proliferation in electrochemical sensing and SERS activity. Trac Trends Anal Chem 2017;86:155-71.

[73] Kailimai S, Ya Z, Shaona C, Shan Z, Yingshuang H, Jiacheng $D$, et al. Selectively encapsulating Ag nanoparticles on the surface of two-dimensional graphene for surface-enhanced Raman scattering. Appl Surf Sci 2019;492: 108-15.

[74] Zhang X, Zhang J, Li W, Yang Y, Qin P, Zhang X, et al. Magnetic graphene oxide nanocomposites as the adsorbent for extraction and pre-concentration of azo dyes in different food samples followed by high-performance liquid chromatography analysis. Food Addit Contam 2018;35: 2099-110.

[75] Carocho M, Morales P, Ferreira IC. Antioxidants: reviewing the chemistry, food applications, legislation and role as preservatives. Trends Food Sci Technol 2018;71:107-20.

[76] Esimbekova EN, Asanova AA, Deeva AA, Kratasyuk VA. Inhibition effect of food preservatives on endoproteinases. Food Chem 2017;235:294-7.

[77] Fan L, Hao Q, Kan X. Three-dimensional graphite paper based imprinted electrochemical sensor for tertiary butylhydroquinone selective recognition and sensitive detection. Sens Actuators, B 2018;256:520-7.

[78] Alamo LS, Tangkuaram T, Satienperakul S. Determination of sulfite by pervaporation-flow injection with amperometric detection using copper hexacyanoferrate-carbon nanotube modified carbon paste electrode. Talanta 2010;81: 1793-9.

[79] Liu H, Na W, Liu Z, Chen X, Su X. A novel turn-on fluorescent strategy for sensing ascorbic acid using graphene quantum dots as fluorescent probe. Biosens Bioelectron 2017;92:229-33.

[80] Mohammadzadeh-Aghdash H, Akbari N, Esazadeh $\mathrm{K}$ Ezzati Nazhad Dolatabadi J. Molecular and technical aspects on the interaction of serum albumin with multifunctional food preservatives. Food Chem 2019;293:491-8.

[81] Uzer A, Saglam S, Can Z, Ercag E, Apak R. Electrochemical determination of food preservative nitrite with gold nanoparticles/p-aminothiophenol-modified gold electrode. Int J Mol Sci 2016;17:1253.

[82] Chen C, Yuan Z, Chang H-T, Lu F, Li Z, Lu C. Silver nanoclusters as fluorescent nanosensors for selective and sensitive nitrite detection. Anal Methods 2016;8:2628-33.

[83] Wang J, Zhu Y, Grimes CA, Nie Z, Cai Q. Eu,Sm,Mn-doped $\mathrm{CaS}$ nanoparticles with $59.3 \%$ upconversion-luminescence quantum yield: enabling ultrasensitive and facile smartphone-based sulfite detection. Anal Chem 2018;90:8658-64.

[84] Jin M, Huang L, Tang Y, Wang G, Ma Z, Yuan Z, et al. Rapid sulfite screening using nitrobenzofurazan anchored asymmetric naphthorhodamine via electrostatic attraction mediated reaction kinetics. Sens Actuators, B 2019;297: 126748.

[85] Chen SM, Manavalan S, Rajaji U, Govindasamy M, Chen TW, Ajmal Ali M, et al. Determination of the antioxidant propyl gallate in meat by using a screen-printed electrode modified with $\mathrm{CoSe}_{2}$ nanoparticles and reduced graphene oxide. Mikrochim Acta 2018;185:520.

[86] Yue X, Luo X, Zhou Z, Bai Y. Selective electrochemical determination of tertiary butylhydroquinone in edible oils based on an in-situ assembly molecularly imprinted polymer sensor. Food Chem 2019;289:84-94.

[87] Li Y, Chen FZ, Xu YT, Yu WJ, Li HY, Fan GC, et al. Selfassembled peptide nanostructures for photoelectrochemical bioanalysis application: a proof-of-concept study. Anal Chem 2019;91:12606-10.

[88] Wang Q, Ruan YF, Zhao WW, Lin P, Xu JJ, Chen HY. Semiconducting organic-inorganic nanodots heterojunctions: platforms for general photoelectrochemical bioanalysis application. Anal Chem 2018;90:3759-65.

[89] Han F, Song Z, Nawaz MH, Dai M, Han D, Han L, et al. $\mathrm{MoS}_{2} / \mathrm{ZnO}$-heterostructures-based label-free, visible-lightexcited photoelectrochemical sensor for sensitive and selective determination of synthetic antioxidant propyl gallate. Anal Chem 2019;91:10657-62.

[90] Sandeep S, Santhosh AS, Swamy NK, Suresh GS, Melo JS, Chamaraja NA. A biosensor based on a graphene nanoribbon/silver nanoparticle/polyphenol oxidase composite matrix on a graphite electrode: application in the analysis of catechol in green tea samples. New J Chem 2018;42: 16620-9.

[91] Chi M, Zhu Y, Jing L, Wang C, Lu X. Fabrication of oxidaselike polyaniline- $\mathrm{MnO}_{2}$ hybrid nanowires and their sensitive 
colorimetric detection of sulfite and ascorbic acid. Talanta 2019;191:171-9.

[92] Ji D, Du Y, Meng H, Zhang L, Huang Z, Hu Y, et al. A novel colorimetric strategy for sensitive and rapid sensing of ascorbic acid using cobalt oxyhydroxide nanoflakes and 3, 3', 5, 5'-tetramethylbenzidine. Sens Actuators, B 2018;256: $512-9$.

[93] Xiong C, Wang Y, Qu H, Zhang L, Qiu L, Chen W, et al. Highly sensitive detection of gallic acid based on organic electrochemical transistors with poly (diallyldimethylammonium chloride) and carbon nanomaterials nanocomposites functionalized gate electrodes. Sens Actuators, B 2017;246:235-42.

[94] Ng KL, Tan GH, Khor SM. Graphite nanocomposites sensor for multiplex detection of antioxidants in food. Food Chem 2017;237:912-20.

[95] Yang H, Lu F, Sun Y, Yuan Z, Lu C. Fluorescent gold nanocluster-based sensor array for nitrophenol isomer discrimination via an integration of host-guest interaction and inner filter effect. Anal Chem 2018;90:12846-53.

[96] Yuan Z, Du Y, Tseng Y-T, Peng M, Cai N, He Y, et al. Fluorescent gold nanodots based sensor array for proteins discrimination. Anal Chem 2015;87:4253-9.

[97] Bordbar MM, Hemmateenejad B, Tashkhourian J, NamiAna SF. An optoelectronic tongue based on an array of gold and silver nanoparticles for analysis of natural, synthetic and biological antioxidants. Microchim Acta 2018;185:493.

[98] Xie Y, Chen T, Guo Y, Cheng Y, Qian H, Yao W. Rapid SERS detection of acid orange II and brilliant blue in food by using $\mathrm{Fe}_{3} \mathrm{O}_{4} @ \mathrm{Au}$ core-shell substrate. Food Chem 2019; 270:173-80.

[99] Zhang D, You H, Yuan L, Hao R, Li T, Fang J. Hydrophobic slippery surface-based surface-enhanced Raman spectroscopy platform for ultrasensitive detection in food safety applications. Anal Chem 2019;91:4687-95.

[100] Mahmoudi-Moghaddam H, Tajik S, Beitollahi H. Highly sensitive electrochemical sensor based on $\mathrm{La}^{3+}$-doped $\mathrm{Co}_{3} \mathrm{O}_{4}$ nanocubes for determination of Sudan I content in food samples. Food Chem 2019;286:191-6.

[101] Heydari M, Ghoreishi SM, Khoobi A. Response surface modeling of electrochemical data for sensitive determination of Sudan III in food products at the surface of a nanocomposite modified electrode. Food Anal Methods 2019;12:1781-90.

[102] Ye Q, Chen X, Yang J, Wu D, Ma J, Kong Y. Fabrication of $\mathrm{CuO}$ nanoparticles-decorated 3D N-doped porous carbon as electrochemical sensing platform for the detection of Sudan I. Food Chem 2019;287:375-81.

[103] Zhao Y, Yamaguchi Y, Liu C, Li M, Dou X. Rapid and quantitative detection of trace Sudan black B in dyed black rice by surface-enhanced Raman spectroscopy (SERS). Spectrochim Acta Mol Biomol Spectrosc 2019;216:202-6.

[104] Hu Y, Gao Z. Sensitive detection of Sudan dyes using tirederived carbon dots as a fluorescent sensor. Spectrochim Acta Mol Biomol Spectrosc 2020;239:118514.

[105] Yao D, Liang A, Jiang Z. A fluorometric clenbuterol immunoassay using sulfur and nitrogen doped carbon quantum dots. Microchim Acta 2019;186:323.

[106] Zhang L, Wang Q, Qi Y, Li L, Wang S, Wang X. An ultrasensitive sensor based on polyoxometalate and zirconium dioxide nanocomposites hybrids material for simultaneous detection of toxic clenbuterol and ractopamine. Sens Actuators, B 2019;288:347-55.

[107] Sun Y, Chen H, Ma P, Li J, Zhang Z, Shi H, et al. In situ synthesis of graphene oxide/gold nanocomposites as ultrasensitive surface-enhanced Raman scattering substrates for clenbuterol detection. Anal Bioanal Chem 2020;412: 193-201.

[108] Shellaiah M, Simon T, Venkatesan P, Sun KW, Ko F-H, $\mathrm{Wu}$ S-P. Nanodiamonds conjugated to gold nanoparticles for colorimetric detection of clenbuterol and chromium(III) in urine. Microchim Acta 2017;185:74.

[109] Peng T, Wang J, Zhao S, Xie S, Yao K, Zheng P, et al. A fluorometric clenbuterol immunoassay based on the use of organic/inorganic hybrid nanoflowers modified with gold nanoclusters and artificial antigen. Microchim Acta 2018;185:366

[110] Li Q, Wang H, Yue X, Du J. Perovskite nanocrystals fluorescence nanosensor for ultrasensitive detection of trace melamine in dairy products by the manipulation of inner filter effect of gold nanoparticles. Talanta 2020;211: 120705.

[111] Nie B, Luo Y, Shi J, Gao L, Duan G. Bowl-like pore array made of hollow Au/Ag alloy nanoparticles for SERS detection of melamine in solid milk powder. Sens Actuators, B 2019;301:127087.

[112] Ren Q, Shen X, Sun Y, Fan R, Zhang J. A highly sensitive competitive immunosensor based on branched polyethyleneimine functionalized reduced graphene oxide and gold nanoparticles modified electrode for detection of melamine. Food Chem 2020;304:125397.

[113] Wang M, Zhao W, Lei S, Zou W, Hu W, Liu H, et al. Rational design of $\mathrm{MnO}$ nanoparticles assemblies for sensitive magnetic relaxation detection of melamine. Talanta 2020;208:120359.

[114] Zhang CJ, Gao ZY, Wang QB, Zhang X, Yao JS, Qiao CD, et al. Highly sensitive detection of melamine based on the fluorescence resonance energy transfer between conjugated polymer nanoparticles and gold nanoparticles. Polymers 2018;10:873. 